\title{
O Papel dos Testes de Estimulação \\ Farmacológica no Diagnóstico da Deficiência de Hormona do Crescimento em Crianças e Adolescentes
}

\author{
Provocative Tests in the Diagnosis of Childhood Onset Growth Hormone \\ Insufficiency
}

Jean-Pierre GONÇALVES ${ }^{1,4}$, Filipa CORREIA ${ }^{2}$, Helena CARDOSO ${ }^{3}$, Teresa BORGES ${ }^{3}$, Maria João OLIVEIRA ${ }^{3}$

Acta Med Port 2014 Sep-Oct;27(5):587-592

\section{RESUMO}

Introdução: A incidência da deficiência de hormona do crescimento é de 1:4000 a 1:10000, sendo a principal indicação para tratamento com hormona do crescimento recombinante.

Objectivos: Avaliar os resultados dos testes de estimulação da hormona do crescimento e identificar factores preditivos para o diagnóstico da deficiência de hormona do crescimento.

Material e Métodos: Estudo observacional, analítico e transversal. Foram analisados dados clínicos e auxológicos e os resultados dos exames de diagnóstico de crianças e adolescentes submetidos a testes de estimulação farmacológica da hormona do crescimento (01/01/2008 a 31/05/2012). O diagnóstico definitivo de deficiência de hormona do crescimento foi efectuado mediante dois testes com estímulos farmacológicos diferentes negativos (pico máximo da hormona do crescimento $<7 \mathrm{ng} / \mathrm{mL}$ ) ou um teste negativo associado à presença de alterações anatómicas da região hipotálamo-hipofisária, observadas na ressonância magnética cerebral. Para análise estatística, foram realizados o testes de t student, do qui-quadrado, correlação de Pearson e a regressão logística. Foi considerado como nível de significância estatística $(p)$ um valor igual ou menor que 0,05 .

Resultados: Realizaram-se testes de estimulação em 89 doentes, com mediana de idade igual a 10 [3-17] anos, 67\% do sexo masculino e $77 \%$ pré-púberes. Os fármacos utilizados no primeiro teste de estimulação foram a clonidina $(n=85)$ e a insulina $(n=4)$. Foram diagnosticados 22 casos de deficiência de hormona do crescimento. Nos casos submetidos a dois testes, os valores máximos de hormona do crescimento apresentaram uma correlação moderada entre si $(r=0,593, p=0,01)$. Verificou-se que as variáveis estatura (z-score) e pico máximo de hormona do crescimento obtido no primeiro teste têm valor preditivo no diagnóstico de deficiência de hormona do crescimento.

Discussão: A determinação do IGF-1 não demonstrou ser preditor de deficiência de hormona do crescimento.

Conclusão: Os testes de estimulação são uma ferramenta de diagnóstico da deficiência de hormona do crescimento e que devem ser enquadrados nos parâmetros clínicos e auxológicos.

Palavras-chave: Alterações do Crescimento; Hormona do Crescimento Humano.

\section{ABSTRACT}

Introduction: The incidence of short stature associated with growth hormone deficiency has been estimated to be about 1:4000 to $1: 10000$. It is the main indication for treatment with recombinant growth hormone.

Objectives: The aims of the study were to evaluate the results of growth hormone stimulation tests and identify the growth hormone deficiency predictors.

Material and Methods: A cross-sectional, analytical and observational study was conducted. We studied all the children and adolescents submitted to growth hormone pharmacological stimulation tests between January 2008 and May 2012. Growth hormone deficiency diagnosis was confirmed by two negatives growth hormone stimulation tests (growth hormone peak $<7 \mathrm{ng} / \mathrm{ml}$ ). The statistical analysis was performed using student t-test, chi-square, Pearson correlation and logistic regression. Statistical significance determined at the $5 \%$ level $(p \leq 0.05)$.

Results: Pharmacological stimulation tests were performed in 89 patients, with a median age of 10 [3-17] years. Clonidine $(n=85)$ and insulin tolerance test $(n=4)$ were the first growth hormone stimulation tests performed. Growth hormone deficiency was confirmed in 22 cases. In cases with two growth hormone stimulation tests, the growth hormone peak showed a moderate correlation $(r=0.593$, $p=0.01$ ). In logistic regression model height (z-score) and the growth hormone peak in first stimulation test were predictors of growth hormone deficiency diagnosis (each one unit increase in z-score decrease the growth hormone deficiency probability).

Discussion: Measurement of IGF-1 cannot be used in diagnosing growth hormone deficiency.

Conclusion: Auxological criteria associated with a positive test seems to be a reliable diagnostic tool for growth hormone deficiency.

Keywords: Growth Disorders; Human Growth Hormone/blood.

\section{INTRODUÇÃo}

A hormona do crescimento $(\mathrm{HC})$ é segregada pelas células somatotróficas da hipófise anterior para a circulação sistémica promovendo o crescimento longitudinal pós-natal,

a regulação do metabolismo lipídico, da aposição óssea, do crescimento da massa muscular esquelética e cardíaca e da tensão arterial. A HC estimula a produção do insulin-like

1. Serviço de Pediatria. Hospital de Braga. Braga. Portugal.

2. Serviço de Pediatria. Hospital do Alto Ave. Guimarães. Portugal.

3. Unidade de Endocrinologia Pediátrica. Centro Hospitalar do Porto. Porto. Portugal.

4. ICVS/3B's Laboratório Associado. Instituto de Investigação em Ciências de Saúde. Escola de Ciências da Saúde. Universidade do Minho. Braga. Portugal.

Recebido: 26 de Setembro de 2013 - Aceite: 13 de Janeiro de 2014 | Copyright @ Ordem dos Médicos 2014 
growth factor (IGF-1) a nível hepático, onde é libertado para a circulação sistémica e localmente em tecidos periféricos, tais como osso, cartilagem e músculo, onde actua de forma parácrina. O IGF-1 circula quase totalmente na sua forma ligada, sendo a insulin-like growth factor binding protein 3 (IGFBP-3) o seu principal ligando ${ }^{1,2}$ e dependente da HC. ${ }^{3}$

Alterações a nível cerebral, hipotalâmico ou hipofisário podem resultar numa deficiência de $\mathrm{HC}$.

A incidência da deficiência de hormona do crescimento (DHC) é de 1:4000 a 1:10000, sendo a principal indicação para tratamento com hormona do crescimento $(\mathrm{HC})$ recombinante. ${ }^{4}$

A DHC pode ser classificada como grave/moderada ou completa/incompleta. ${ }^{5,6} \mathrm{Na}$ ausência de um método gold standard, o diagnóstico é baseado em parâmetros clínicos, auxológicos, analíticos e radiológicos. ${ }^{7}$

Apesar de não existir consenso relativo ao seu valor preditivo, os testes de estimulação continuam na atualidade a serem utilizados para confirmação do diagnóstico da DHC e para a tomada de decisão para iniciar terapêutica com HC recombinante. ${ }^{7,8}$ São necessárias duas provas com estímulos diferentes com valores máximos de $\mathrm{HC}$ inferiores a $7 \mathrm{ng} / \mathrm{ml}$ para confirmar secreção insuficiente de HC. ${ }^{9-11}$ No presente estudo os autores pretendem avaliar os resultados dos testes de estimulação da secreção da $\mathrm{HC}$, nomeadamente a concordância entre os picos de secreção da $\mathrm{HC}$ perante dois estímulos farmacológicos diferentes e identificar factores preditivos para deficiência de $\mathrm{HC}$ nos casos em que se confirmou o diagnóstico após a realização dos testes de estimulação.

\section{MATERIAL E MÉTODOS}

Estudo observacional, transversal e analítico de crianças e adolescentes submetidos a testes de estimulação farmacológica da secreção da HC, na Unidade de Endocrinologia Pediátrica do Centro Hospitalar do Porto, no período de Janeiro de 2008 a Maio de 2012.

A recolha de informação foi efectuada através da análise retrospectiva dos registos clínicos. Foram analisados os parâmetros relativos à estatura, índice de massa corporal (IMC), velocidade de crescimento (VC), estatura alvo familiar (EAF), pico da HC (ng/ml), IGF-1, IGFBP-3, idade óssea (IO) e ressonância magnética nuclear (RMN) cerebral, se aplicável. A estatura, IMC, VC e EAF foram padronizados, atendendo à idade e género e representados por z-scores. $\mathrm{O}$ z-score da estatura, EAF e VC foram determinados e interpretados usando as curvas de Tanner-Whitehouse..$^{12,13}$ $\mathrm{O}$ z-score do IMC foi avaliado pelas curvas de Cole. ${ }^{14} \mathrm{O}$ IGF-1 e IGFBP-3 foram expressos dicotomicamente usando como valor de referência o percentil 2,5, para idade cronológica e género (Immulite 2000; Diagnostic Products Corp., Los Angeles, CA).$^{15} \mathrm{~A}$ idade óssea foi determinada pelo método de Greulich e Pyle. ${ }^{16}$

Em concordância com as normas da Comissão Nacional de Normalização da Hormona do Crescimento (CN$\mathrm{NHC}$ ), um pico máximo de $\mathrm{HC}$ após estímulo farmocológico adequado $<7 \mathrm{ng} / \mathrm{ml}$ foi considerado como indicativo de deficiência de secreção $\mathrm{HC}^{11}$. Os testes de estimulação farmacológica foram classificados como negativos se pico máximo de $\mathrm{HC}<7 \mathrm{ng} / \mathrm{ml}$ e positivos se pico máximo de $\mathrm{HC} \geq 7 \mathrm{ng} / \mathrm{ml}$. O diagnóstico de DHC foi confirmado após a obtenção de dois testes com estímulos farmacológicos diferentes negativos.

Foram definidos dois grupos de estudo: grupo 1 (casos com dois testes negativos) e grupo 2 (casos com um teste negativo e outro positivo)

A descrição dos resultados foi efectuada através de medidas de dispersão (média \pm DP). Na comparação das variáveis por grupos ( 1 e 2 ) foi utilizado o teste $t$-student nas variáveis quantitativas e o teste do qui-quadrado nas variáveis qualitativas.

A correlação entre os picos de $\mathrm{HC}$ observados no primeiro e segundo teste foi determinada através da correlação de Pearson.

$\mathrm{Na}$ análise de possíveis preditores de DHC, confirmada por dois testes negativos, recorreu-se à regressão logística. Foram definidos dois modelos, um com a variável IGF-1 (modelo 1) e um modelo com IGF-1 ajustado para estatura e pico de $\mathrm{HC}$ do primeiro teste (modelo 2).

Os dados foram analisados através do programa informático SPSS â (versão 19.0). Foram considerados resultados com significado estatístico para $p<0,05$.

\section{RESULTADOS}

Na figura 1 está representada a distribuição dos casos por estímulo farmacológico utilizado e número de testes efectuados. No total foram efectuadas provas de estimulação em 89 doentes, com mediana de idade igual a 10 [3-17] anos, $67 \%$ do sexo masculino e $77 \%$ com estádio pubertário I.

No primeiro teste de estimulação foi utilizada a clonidina $(n=85)$ e a insulina $(n=4)$.

Da análise dos primeiros testes, 29 doentes tiveram um teste negativo. Destes, 24 foram submetidos a segundo teste [clonidina $(n=2)$, hipoglicemia-insulínica $(n=5)$, L-dopa $(n=17)]$.

Dos 24 submetidos a segundo teste, 22 deles tiveram um resultado negativo, o que permitiu confirmar a secreção insuficiente de $\mathrm{HC}$ e o diagnóstico de DHC.

Em cinco casos, apesar de primeiro teste negativo, não foi efectuada um segundo teste pelos seguintes motivos: perda de seguimento $(n=1)$, Síndrome de Silver Russel $(n=1)$, Síndrome de Kallman $(n=1)$ e criança com alteração do eixo hipotálamo-hipófise $(n=2)$ na RMN cerebral.

Dos 60 doentes em que foi excluída DHC após realização do primeiro teste:

a) Um total de 33 apresentava z-score para estatura < -2 SDS (10 com critérios de atraso constitucional do crescimento e maturação e 17 com critérios de baixa estatura familiar);

b) Um total de 27 apresentava um z-score para estatura $\geq-2$, e um dos seguintes critérios: $z$-score da estatura < $-1,5$ em relação EAF, $z$-score da $\mathrm{VC}<-2$ durante um ano $\mathrm{e}$ $z$-score da $\mathrm{VC}<-1,5$ durante dois anos. 


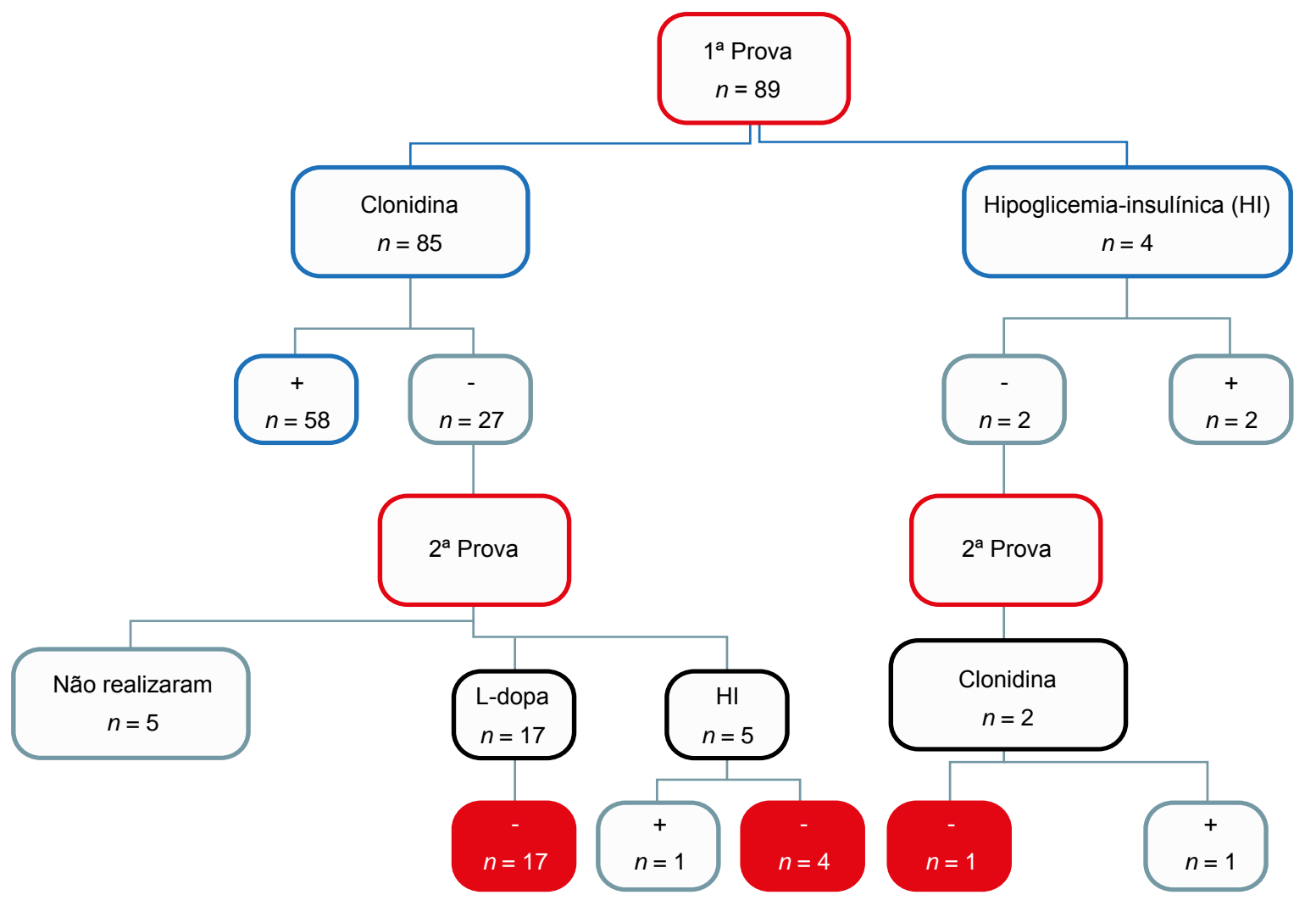

Figura 1 - Distribuição dos casos por tipo e número de testes de estimulação

+ teste de estimulação positivo (pico $\mathrm{HC} \geq 7 \mathrm{ng} / \mathrm{ml}$ ); - teste de estimulação negativo (pico $\mathrm{HC}<7 \mathrm{ng} / \mathrm{ml}$ )

Em todos os casos foi avaliado o IGF-1 e a idade óssea, em 35 casos o IGF BP3 e 32 indivíduos efectuaram RMN cerebral.

Os doentes com DHC (grupo1) apresentavam, comparativamente com os restantes casos (grupo 2), média de estatura (z-score) $(-2,9 \pm 0,6$ vs $-2,1 \pm 0,9, p=0,001)$ e um pico máximo de $\mathrm{HC}$ no primeiro teste $(4,0 \pm 2,1$ vs $16,1 \pm$ $6,5, p=0,001$ ) inferiores (Tabela 1 ), com valores estatisticamente significativos. A proporção de doentes com valores de IGF-1 e IGF BP3 inferiores ao percentil 2,5 (para a idade cronológica) foi significativamente superior no grupo 1 ([IGF- $1(54,5 \%$ vs $26,2 \%, p=0,016)]$, [IGF BP3 $(30,0 \%$ vs $0 \%, p=0,007)]$ ) (Tabela 1).

Nos casos submetidos a dois testes, os valores máximos de $\mathrm{HC}$ apresentaram uma correlação moderada entre si $(r=0,593, p=0,01)$ (Fig. 2).

No modelo 1 de regressão logística verificou-se que os casos com IGF-1 inferior ao percentil 2,5 tinham uma probabilidade de ter DHC 3,4 vezes superior comparativamente aos casos com IGF-1 superior ao percentil 2,5 (Tabela 2). No entanto, no modelo 2, ajustado para estatura (z-score) e pico de HC, o IGF1 não apresentou valor preditivo. A estatura (z-score) e o pico de $\mathrm{HC}$ no primeiro teste apresentavam valor preditivo no diagnóstico de $\mathrm{DHC}$, isto é, à medida que aumentam diminui a probabilidade de DHC (Tabela 2).

\section{DISCUSSÃO}

O diagnóstico da $\mathrm{DHC}$ em crianças constitui frequentemente um verdadeiro desafio clínico ${ }^{7}$ e a certeza do diag- nóstico em muitos casos só pode ser afirmado após ter sido atingida a estatura final e efectuada uma reavaliação da secreção da HC.

Para confirmação da suspeita de DHC vários métodos de diagnóstico tem sido sugeridos, entre eles os testes fisiológicos e farmacológicos, de estimulação da secreção da HC, $, 5,6,17$ o IGF-1 e o IGFBP-3..$^{18,19}$

Os testes farmacológicas de estimulação, apesar das limitações conhecidas (estímulo não fisiológico, limites de cut-off arbitrários, variabilidade intra-individual na resposta ao fármaco), ${ }^{20,21}$ continuam a respresentar o método mais consensual para avaliação da secreção da $\mathrm{HC} .{ }^{22}$

Duas provas com estímulos diferentes com pico da $\mathrm{HC}$ $<10 \mathrm{ng} / \mathrm{ml}$, em crianças com BE, sustentam o diagnóstico da DHC. ${ }^{8}$ Contudo, atendendo ao facto de existir um espectro desde secreção normal da $\mathrm{HC}$ até ausência total da $\mathrm{HC}$ a definição de um cut-off específico para DHC é difícil e arbitrária. ${ }^{23}$ Cianfarani e os seus colaboradores demonstraram que a diminuição do cut-off dos $10 \mathrm{ng} / \mathrm{ml}$ para $7 \mathrm{ng} /$ $\mathrm{ml}$ aumenta significativamente a especificidade e diminui a sensibilidade. ${ }^{23} \mathrm{~A}$ CNNHC nas suas recomendações estabelece que, nos casos de défice idiopático da $\mathrm{HC}$, duas provas de estimulação com pico da $\mathrm{HC}<7 \mathrm{ng} / \mathrm{ml}$ sustentam o diagnóstico e suportam o início da terapêutica com $\mathrm{HC}$ recombinante. ${ }^{11}$

Nesta população em estudo o teste de estimulação com clonidina oral foi o mais utilizado, sendo o fármaco utilizado no primeiro teste em $96 \%$ dos doentes. Este fármaco, a adrenérgico, promove a estimulação da secreção da $\mathrm{HC}^{24,25}$ 
Tabela 1 - Análise descriptiva das crianças e adolescentes submetidos a testes de estimulação com (Grupo1) e sem (Grupo 2) confirmação de DHC

\begin{tabular}{|c|c|c|c|c|}
\hline & & $\begin{array}{c}\text { Grupo } 1 \\
n=22\end{array}$ & $\begin{array}{c}\text { Grupo } 2 \\
n=62\end{array}$ & \\
\hline & & $n(\%)$ & $n(\%)$ & valor de $p$ \\
\hline Sexo & masculino & $13(59,1)$ & $44(71,0)$ & 0,305 \\
\hline \multirow[t]{5}{*}{ Estádio Pubertário } & I & $15(71,4)$ & $39(79,0)$ & 0,717 \\
\hline & II & $5(23,8)$ & $7(14,3)$ & \\
\hline & III & $1(4,8)$ & $2(4,1)$ & \\
\hline & IV & $0(0)$ & $0(0)$ & \\
\hline & V & $0(0)$ & $1(2,0)$ & \\
\hline IGF1 & $<\mathrm{P} 2,5$ & $12(54,5)$ & $16(26,2)$ & 0,016 \\
\hline IGF BP3 & $<\mathrm{P} 2,5$ & $3(30,0)$ & $0(0)$ & 0,007 \\
\hline \multirow[t]{2}{*}{$\Delta \mathrm{IC} / \mathrm{IO}$} & IC-IO > 24 meses & $10(45,5)$ & $17(27,4)$ & 0,120 \\
\hline & & Média (DP) & Média (DP) & valor de $p$ \\
\hline Idade & anos & $9,4(3,3)$ & $9,7(3,0)$ & 0,683 \\
\hline Estatura & z-score & $-2,87(0,6)$ & $-2,09(0,9)$ & 0,001 \\
\hline Estatura (ajustada EAF) & z-score & $-1,50(1,0)$ & $-0,99(1,1)$ & 0,055 \\
\hline VC & z-score & $-2,22(1,6)$ & $-1,26(2,4)$ & 0,067 \\
\hline IMC & z-score & $-0,23(1,1)$ & $-0,21(2,9)$ & 0,944 \\
\hline Pico de HC (1 ${ }^{a}$ prova $)$ & $\mathrm{ng} / \mathrm{mL}$ & $4,0(2,1)$ & $16,1(6,5)$ & 0,001 \\
\hline$\Delta \mathrm{IC} / \mathrm{IO}$ & meses & $22,7(15,7)$ & $15,7(17,2)$ & 0,099 \\
\hline
\end{tabular}

DP: desvio padrão; IGF1: insulin growth factor 1; IGF BP3: insulin growth factor binding protein 3; IC: idade cronológica; IO: idade óssea; HC: hormona do crescimento; EAF: estatura alvo familiar; VC: velocidade crescimento; IMC: índice de massa corporal

e é dependente da idade, ${ }^{26}$ obesidade e actividade física. ${ }^{27}$ O valor preditivo positivo de um teste único com clonidina na confirmação de DHC é de $80 \%$ usando como referência o valor máximo de $\mathrm{HC}$ igual a $10 \mathrm{ng} / \mathrm{ml} .^{8}$

Dos 24 casos submetidos a dois testes, a clonidina foi o fármaco utilizado no primeiro teste em 22 casos. Apenas um caso com primeiro teste de clonidina negativa $(<7 \mathrm{ng} /$ $\mathrm{ml}$ ) apresentou segunda prova positiva (hipoglicemia insulinica). O segundo caso com segundo teste positivo, fez como primeiro teste da hipoglicemia-insulínica e o segundo com clonidina. Salienta-se o facto, que nestes dois casos com segundo teste positivo, o pico da HC foi inferior ao valor de referência considerado por diversos autores como secreção normal $(10 \mathrm{ng} / \mathrm{ml}){ }^{8}$

A confirmação da $\mathrm{DHC}$ geralmente implica a realização de dois testes farmacológicos com pico de $\mathrm{HC}$ inferior a $7 \mathrm{ng} / \mathrm{ml} .{ }^{11}$ No entanto, nos casos com critérios de DHC e uma etiologia conhecida (malformações do sistema nervoso central e genéticos), é suficiente apenas um teste de estimulação. ${ }^{7,11,28} \mathrm{Em}$ quatro dos cinco casos em que a etiologia para $\mathrm{DHC}$ era conhecida (com primeiro teste de esti- mulação negativo) não se realizou segunda prova.

Apesar da determinação do IGF-1 e IGFBP-3 em crianças e adolescentes ser considerada útil na confirmação do diagnóstico da $\mathrm{DHC},{ }^{29-31}$ a sua utilidade e valor preditivo não são consensuais. ${ }^{23,32}$ Estudos nesta área concluiram que ambos os parâmetros não descriminavam as crianças com BEI daquelas com DHC. ${ }^{23}$ No presente estudo o valor do IGF-1, quando ajustado para estatura e valor de pico da $\mathrm{HC}$ no primeiro teste, não apresentou um valor preditivo no diagnóstico da DHC.

Os parâmetros que apresentaram valor preditivo da DHC, confirmada por dois testes de estimulação negativa, foram a estatura (z-score) e o pico da HC no primeiro teste. Como anteriormente mencionado, todos os casos com primeira prova negativa, apresentaram na segunda prova um valor de pico da $\mathrm{HC}<10 \mathrm{ng} / \mathrm{ml}$. Assim, parece razoável sugerir que os critérios auxológicos associados a um teste negativo, possam ser suficientes para o diagnóstico de DHC. A importância da auxologia no diagnóstico da DHC, é levada ao extremo, por exemplo na Austrália, em que a selecção dos doentes para tratamento com $\mathrm{HC}$ recombinante 


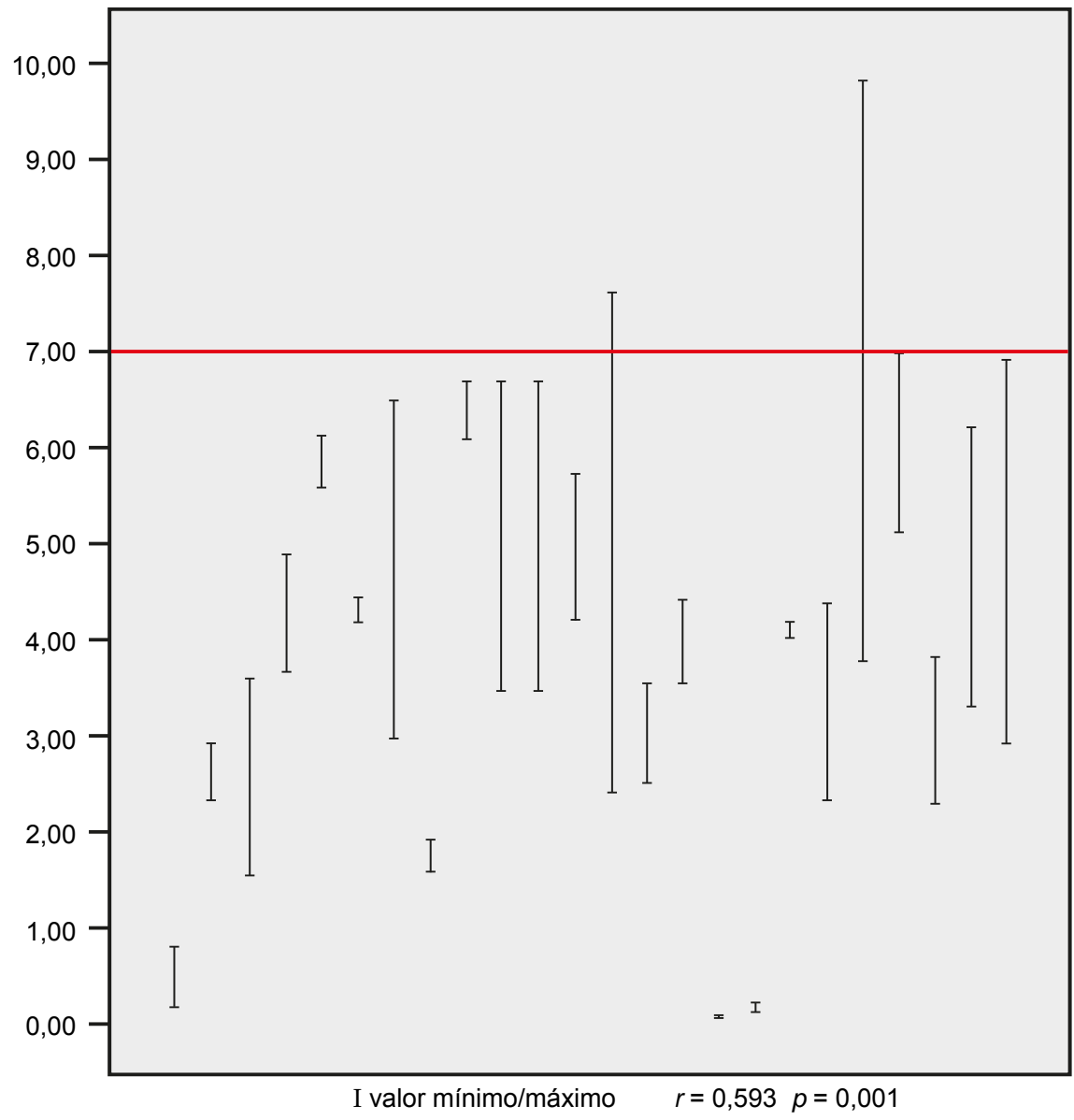

Figura 2 - Variação do pico de $\mathrm{HC}(\mathrm{ng} / \mathrm{ml})$ nos casos submetidos a dois testes de estimulação

Tabela 2 - Preditores de DHC confirmada por testes de estimulação

\begin{tabular}{cccc}
\hline Modelo & Variáveis & OR [IC95\%] & Valor de $\boldsymbol{p}$ \\
\hline 1 & IGF1 $(<\mathrm{P} 2,5)$ & $3,4[1,2-9,3]$ & $\mathbf{0 , 0 1 9}$ \\
2 & IGF1 $(<\mathrm{P} 2,5)$ & $1,1[0,1-16,0]$ & 0,954 \\
& Estatura (z-score) & $0,1[0,1-0,8]$ & $\mathbf{0 , 0 3 6}$ \\
\hline
\end{tabular}

é baseada apenas em parâmetros auxológicos. ${ }^{33,34}$ Contudo, um estudo norte-americano demonstrou não existir diferenças na estatura, índice de massa corporal e velocidade de crescimento em crianças com BEl e deficiência de hormona do crescimento. ${ }^{35}$

\section{CONCLUSÃO}

Os testes de estimulação são uma ferramenta de diagnóstico da DHC e devem ser enquadrados nos parâmetros clínicos e auxológicos.

\section{CONFLITOS DE INTERESSE}

Os autores declaram a inexistência de quaisquer conflitos de interesse.

\section{FONTE DE FINANCIAMENTO}

Os autores declaram que o presente trabalho não foi objecto de quaisquer financiamentos. 
1. Rosenfeld RG, Lamson G, Pham H, Oh Y, Conover C, De Leon DD, et al. Insulin like growth factor-binding proteins. Recent Prog Horm Res. 1990;46:99-159; discussion 159-63

2. Baxter RC, Martin JL. Binding proteins for the insulin-like growth factors: structure, regulation and function. Prog Growth Factor Res. 1989;1:4968.

3. Baxter RC, Martin JL. Radioimmunoassay of growth hormonedependent insulin-like growth factor binding protein in human plasma. J Clin Invest. 1986;78:1504-12.

4. Rona RJ, Tanner JM. Aetiology of idiopathic growth hormone deficiency in England and Wales. Arch Dis Child. 1977;52:197-208.

5. Rosenfeld RG, Albertsson-Wikland K, Cassorla F, Frasier SD, Hasegawa Y, Hintz RL, et al. Diagnostic controversy: the diagnosis of childhood growth hormone deficiency revisited. J Clin Endocrinol Metab. 1995;80:1532-40.

6. Shalet SM, Toogood A, Rahim A, Brennan BM. The diagnosis of growth hormone deficiency in children and adults. Endocr Rev. 1998;19:203-23.

7. GH Research Society. Consensus guidelines for the diagnosis and treatment of growth hormone $(\mathrm{GH})$ deficiency in childhood and adolescence: summary statement of the GH Research Society. J Clin Endocrinol Metab. 2000;85:3990-3.

8. Ranke MB. Diagnosis of growth hormone deficiency and growth hormone stimulation tests. In: Diagnostics of endocrine function in children and adolescents. $3^{\text {rd }}$ edition. Basel: S. Karger; 2003. p.107-28.

9. The Health Services Human Growth Hormone Committee. Comparison of the intravenous insulin and oral clonidine tolerance tests for growth hormone secretion. Arch Dis Child. 1981;56:852-4.

10. Rahim A, Toogood AA, Shalet SM. The assessment of growth hormone status in normal young adult males using a variety of provocative agents. Clin Endocrinol. 1996;45:557-62.

11. Comissão Nacional de Normalização da Hormona do Crescimento (CNNHC). Avaliação de crianças e adolescentes com baixa estatura Lisboa: Ministério da Saúde;2004.

12. Tanner JM, Whitehouse RH. Clinical longitudinal standards for height, weight, height velocity, weight velocity, and stages of puberty. Arch Dis Child. 1976;51:170-9

13. Tanner JM, Whitehouse RH, Takaishi M. Standards from birth to maturity for height, weight, height velocity, and weight velocity: British children, 1965. II. Arch Dis Child. 1966;41:613-35.

14. Cole TJ. A chart to link child centiles of body mass index, weight and height. Eur J Clin Nutr. 2002;56:1194-9.

15. Elmlinger MW, Kuhnel W, Weber MM, Ranke MB. Reference ranges for two automated chemiluminescent assays for serum insulin-like growth factor I (IGF-I) and IGF-binding protein 3 (IGFBP-3). Clin Chem Lab Med. 2004;42:654-64.

16. Greulich SI Radiographic atlas of skeletal development of the hand and wrist. $2^{\text {nd }}$ edition. Stanford: Stanford University Press; 1959.

17. Ghigo E, Bellone J, Aimaretti G, Bellone S, Loche S, Cappa M, et al. Reliability of provocative tests to assess growth hormone secretory status. Study in 472 normally growing children. J Clin Endocrinol Metab. 1996;81:3323-7.

18. Blum WF, Albertsson-Wikland K, Rosberg S, Ranke MB. Serum levels of insulin-like growth factor I (IGF-I) and IGF binding protein 3 reflect spontaneous growth hormone secretion. J Clin Endocr Metabolism. 1993;76:1610-6.
19. Blum WF, Ranke MB, Kietzmann K, Gauggel E, Zeisel HJ, Bierich JR A specific radioimmunoassay for the growth hormone $(\mathrm{GH})$-dependent somatomedin-binding protein: its use for diagnosis of $\mathrm{GH}$ deficiency. J Clin Endocr Metab. 1990;70:1292-8.

20. Cacciari E, Tassoni P, Parisi G, Pirazzoli P, Zucchini S, Mandini M, et al. Pitfalls in diagnosing impaired growth hormone $(\mathrm{GH})$ secretion: retesting after replacement therapy of 63 patients defined as $\mathrm{GH}$ deficient. J Clin Endocrinol Metab. 1992;74:1284-9.

21. Nicolson A, Toogood AA, Rahim A, Shalet SM. The prevalence of severe growth hormone deficiency in adults who received growth hormone replacement in childhood. Clin Endocrinol. 1996;44:311-6.

22. Rose SR, Ross JL, Uriarte M, Barnes KM, Cassorla FG, Cutler GB, Jr The advantage of measuring stimulated as compared with spontaneous growth hormone levels in the diagnosis of growth hormone deficiency. $\mathrm{N}$ Engl J Med. 1988;319:201-7.

23. Cianfarani S, Tondinelli T, Spadoni GL, Scire G, Boemi S, Boscherin B. Height velocity and IGF-I assessment in the diagnosis of childhood onset $\mathrm{GH}$ insufficiency: do we still need a second $\mathrm{GH}$ stimulation test? Clin Endocrinol. 2002;57:161-67.

24. Gil-Ad I, Topper E, Laron Z. Oral clonidine as a growth hormone stimulation test. Lancet. 1979;2:1242.

25. Lal S, Tolis G, Martin SB, Brown GM, Guyda H. Effect of clonidine on growth hormone, prolactin, luteinizing hormone, follicle-stimulating hormone, and thyroid-stimulating hormone in the serum of normal men. J Clin Endocrinol Metab. 1975;41:827-32.

26. Gil-Ad I, Gurewitz R, Marcovici O, Rosenfeld J, Laron Z. Effect of aging on human plasma growth hormone response to clonidine. Mech Ageing Devel. 1984;27:97-100.

27. Vahl N, Jorgensen JO, Jurik AG, Christiansen JS. Abdominal adiposity and physical fitness are major determinants of the age associated decline in stimulated $\mathrm{GH}$ secretion in healthy adults. J Clin Endocrinol Metab. 1996;81:2209-15.

28. Richmond EJ, Rogol AD. Growth hormone deficiency in children Pituitary. 2008;11:115-20.

29. Hasegawa Y, Hasegawa T, Aso T, Kotoh S, Tsuchiya Y, Nose O, et al. Comparison between insulin-like growth factor-I (IGF-I) and IGF binding protein-3 (IGFBP-3) measurement in the diagnosis of growth hormone deficiency. Endocr J. 1993;40:185-90.

30. Nunez SB, Municchi G, Barnes KM, Rose SR. Insulin-like growth factor (IGF-I) and IGF-binding protein-3 concentrations compared to stimulated and night growth hormone in the evaluation of short children-a clinical research center study. J Clin Endocrinol Metab. 1996;81:1927-32.

31. Clemmons DR, Underwood LE. Clinical review 59: Uses of human insulin-like growth factor-I in clinical conditions. J Clin Endocrinol Metab. 1994;79:4-6.

32. Moore DC, Ruvalcaba RH, Smith EK, Kelley VC. Plasma somatomedin-C as a screening test for growth hormone deficiency in children and adolescents. Hormone Res. 1982;16:49-55.

33. Werther GA. Growth hormone measurements versus auxology in treatment decisions: the Australian experience. J Pediatrics. 1996;128:S47-51.

34. Cowell CT, Dietsch S, Greenacre P. Growth hormone therapy for 3 years: the OZGROW experience. J Paediatr Child Health. 1996;32:86-93.

35. Hintz RL. The role of auxologic and growth factor measurements in the diagnosis of growth hormone deficiency. Pediatrics. 1998;102:524-6. 


\section{O Papel dos Testes de Estimulação Farmacológica no Diagnóstico da Deficiência de Hormona do Crescimento em Crianças e Adolescentes}

Acta Med Port 2014:27:587-592

Publicado pela Acta Médica Portuguesa, a Revista Científica da Ordem dos Médicos

Av. Almirante Gago Coutinho, 151

1749-084 Lisboa, Portugal.

Tel: +351 218428215

E-mail: submissao@actamedicaportuguesa.com

www.actamedicaportuguesa.com

ISSN:0870-399X | e-ISSN: 1646-0758

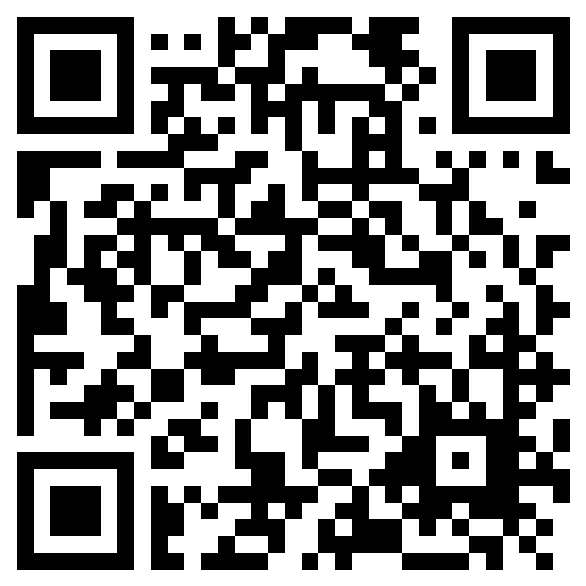

